\title{
Insect Growth Regulators for Insect Pest Control
}

\author{
Sanjay Cyril Masih ${ }^{1 *}$ and Bhat Rayees Ahmad ${ }^{2}$ \\ ${ }^{1}$ Department of Zoology, Ewing Christian Post Graduate College, Allahabad, India \\ ${ }^{2}$ Department of Zoology, Kurukshetra University, Kurukshetra, India \\ *Corresponding author
}

\section{A B S T R A C T}

\section{Keywords}

Insect growth regulators,

Carvacrol,

Derivatives insect

pests, Insect

hormones

\section{Article Info}

Accepted:

04 November 2019

Available Online:

10 December 2019
Insecticides with growth regulating properties (IGR) may adversely affect insects by regulating or inhibiting specific biochemical pathways or processes essential for insect growth and development. Some insects exposed to such compounds may die due to abnormal regulation of hormone-mediated cell or organ development. Other insects may die either from a prolonged exposure at the developmental stage to other mortality factors (susceptibility to natural enemies, environmental conditions etc.) or from an abnormal termination of a developmental stage itself. Insect growth regulators may come from a blend of synthetic chemicals or from other natural sources, such as plants. The chemical composition of hormones indigenous to insects is now being studied and used as a basis for developing analogs or mimics against insects. The similarities, however, in certain aspects of biochemistry among vertebrates and invertebrates may result in the limited development of IGRs. Environmental contamination also creates a hurdle as well as a challenge for industries to develop compounds that provide a more environmentally or ecologically sound insect pest control. As part of on-going search for new biologically active molecules prepared from compound of natural origin, we thought of Insect Growth Regulators as substitute to insecticides that are commercially used.

\section{Introduction}

Insect Growth Regulators (IGRs) are compounds which interfere with the growth, development and metamorphosis of insects. IGRs include synthetic analogues of insect hormones such as ecdysoids and juvenoids and non-hormonal compounds such as precocenes (Anti $\mathrm{JH}$ ) and chitin synthesis inhibitors. Natural hormones of insects which play a role in growth and development are: 1. Brain hormone: They are also called activation hormone $(\mathrm{AH}) . \mathrm{AH}$ is secreted by neuro secretory cells (NSC) which are neurons of central nervous system (CNS). Its role is to activate the corpora allata to produce juvenile hormone (JH). 2. Juvenile hormone (JH): Also called neotinin. It is secreted by corpora allata which are paired glands present behind insect brain. Their role is to keep the larva in 
juvenile condition. JH I, JH II, JH III and JH IV have been identified in different groups of insects. The concentration of $\mathrm{JH}$ decreases as the larva grows and reaches pupal stage. JH I, II and IV are found in larva while JH III is found in adult insects and are important for development of ovary in adult females. 3. Ecdysone: Also called Moulting hormone $(\mathrm{MH})$. Ecdysone is a steroid and is secreted by Prothoracic Glands (PTG) present near prothoracic spiracles. Moulting in insects is brought about only in the presence of ecdysone. Ecdysone level decreases and is altogether absent in adult insects. Although, agricultural pesticides have prevented pest damage of between 5\% and $30 \%$ of potential production in many crops, however, they have posed a number of problems in agriculture, including the killing of beneficial insects, outbreak of secondary pests, and the development of pesticide resistant pests. Insect Growth Regulators (IGR's) disrupt and impede the development of the life cycle of insects in the larval and egg stage. In short, IGR is a form of "birth control" for pests which helps keep the populations of unwanted pests under control by preventing current and future infestations.

\section{IGRs used in pest management}

\section{Ecdysoids}

These compounds are synthetic analogues of natural ecdysone. When applied in insects, kill them by formation of defective cuticle. The development processes are accelerated bypassing several normal events resulting in integument lacking scales or wax layer.

\section{Juvenoids (JH mimics)}

They are synthetic analogues of Juvenile Hormone $(\mathrm{JH})$. They are most promising as hormonal insecticides. JH mimics were first identified by Williams and Slama in the year
1966. They found that the paper towel kept in a glass jar used for rearing a Pyrrhocoris bug caused the bug to die before reaching adult stage. They named the factor from the paper as 'paper factor' or 'juvabione'. They found that the paper was manufactured from the wood pulp of balsam fir tree (Abies balsamea) which contained the JH mimic. Juvenoids have anti-metamorphic effect on immature stages of insect. They retain status quo in insects (larva remains larva) and extra (super numerary) moultings take place producing super larva, larval-pupal and pupal-adult intermediates which cause death of insects. Juvenoids are larvicidal and ovicidal in action and they disrupt diapause and inhibit embryogenesis in insects. Methoprene is a $\mathrm{JH}$ mimic and is useful in the control of larva of hornfly, stored tobacco pests, green house homopterans, red ants, leaf mining flies of vegetables and flowers.

\section{Anti JH or precocenes}

They act by destroying corpora allata and preventing JH synthesis. When treated on immature stages of insect, they skip one or two larval instars and turn into tiny precocious adults. They can neither mate, nor oviposit and die soon. e.g. EMD, FMev, and PB (Piperonyl Butoxide).

\section{Chitin synthesis inhibitors}

Benzoyl phenyl ureas have been found to have the ability of inhibiting chitin synthesis in vivo by blocking the activity of the enzyme chitin synthetase. Two important compounds in this category are Diflubenzuron (Dimilin) and Penfluron. The effects they produce on insects include: Disruption of moulting, Displacement of mandibles and labrum, Adult fails to escape from pupal skin and dies and Ovicidal effect. Chitin synthesis inhibitors have been registered for use in many countries and used successfully against pests of soybean, cotton, 
apple, fruits, vegetables, forest trees and mosquitoes and pests of stored grain

A new approach to insect pest control is the use of substances that adversely affect insect growth and development. These substances are classified as "insect hormone mimics" or “insect growth regulators' (IGRs) owing to their effects on certain physiological regulatory processes essential to the normal development of insects or their progeny. They are quite selective in their mode of action and potentially act only on target species. The action of IGRs, however, should not be confused with other synthetic insecticides, such as organophosphates and carbamates, since these chemicals interfere with other physiological processes but do not regulate the development of normal insects. An IGR, therefore, does not necessarily have to be toxic to its target, but may lead instead to various abnormalities that impair insect survival (Siddall, 1976). Interestingly, most of the IGRs that have shown effectiveness against insect pests cause the rapid death of the insect through failure of a key regulatory process to operate or function. IGRs generally control insects either through regulation of metamorphosis or interference with reproduction (Riddiford and Truman, 1978). Compounds developed to disrupt metamorphosis ensure that no reproductive adults are formed. Those that specifically interfere with reproduction may include the development of adults with certain morphogenetic abnormalities that reduce their reproductive potential. Adults may be sterile or possess abnormally developed genitalia, which hinders the mating process or the capacity to produce fertile offspring. Chitin synthesis inhibitors, the insect cuticle serves as an interface between the living animal and its environment; and forms the exoskeleton, supporting the linings of the gut, respiratory systems, reproductive ducts, and some gland ducts. It consists of protein and chitin fractions. In the 1970, Benzoyl phenyl ureas, compounds with high degree of selectivity and low mammalian toxicity were discovered by scientists at Philips-Duphar, Netherlands. Actually it was a attempt for development of weed control agent but the product was found to be more effective as an insecticide showing delay in toxicity when the insect next molted.

Because CSIs interfere with the polymerization of chitin, this mode of action has been targeted for control of several different insect pests. CSIs cause abnormal deposits of endocuticle that accumulate during molting, specifically uridine diphospho-Nacetylglucosamine monomers thereby preventing chitin synthesis. This produce a weakened cuticle and causes mortality when the pro-cuticle is subjected to the stresses of ecdysis and cuticular expansion. Consequences of CSI toxicity also include mortality in the absence of metamorphosis and include swollen appendages, decrease in locomotion, inability to eat due to dislocation of mandibles, malformed or absent peritrophic matrix, as well as suppressed fecundity and egg viability.

\section{Benzoyl Phenyl Ureas (BPU)}

Benzoyl phenyl urea, an important type of insect growth regulators, acting on the larval stages of the Lepidoptera insects by inhibiting chitin synthesis have been rapidly developed after the introduction of the first benzoyl phenyl urea diflubenzuron in 1972. Besides diflubenzuron, hexaflumuron, lufenuron, penflzuron, noveluron, tefluenzuron and chlorfluazuron were some of other widely used IGRs. Benzoyl phenyl urea have a unique mode of action coupled with a high degree of activity on target pests and low toxicity to non-target organisms, that is why they have attracted considerable attention for decades and have become a new tool for integrated pest management. 
The in vivo or in situ (isolated integument incubated in a tissue culture medium) studies showed that chitin synthetase catalyzing the incorporation of UDPN-acetylglucosamine (UDP NAGA) to chitin was clearly inhibited by BPUs. However, all of the subsequent studies trying to prove some action of BPUs on any part of the chitin synthesis pathway in insects in cell free systems (rather than in vivo and in situ) failed. In addition, BPUs showed no inhibitory actions on fungal chitin synthetase in vivo as well as in cell free systems, which have roughly equivalent chitin synthesis pathways as insects. Therefore, the action mechanism of BPUs remained unanswered.

\section{Juvenile hormone}

Although numerous natural substances regulate the growth and development of insects, the juvenile hormones $(\mathrm{JH})$ have been singled out for refinement of their biological and chemical properties by synthesis of chemical analogs which are insect growth regulators. Historically, the major reasons for the selection of $\mathrm{JH}$ as a rational lead for pesticide design were the beliefs Williams (1967) that this hormone occurred in insects, that it had a specific function in insects, and that it did not occur in higher animals. The implication was that juvenile hormone would therefore be selectively active in insects. Although current knowledge strongly supports the beliefs, we have no formal proof that $\mathrm{JH}$ does not occur outside the class of insects. It may be philosophically impossible to obtain proof since we are only able to say that $\mathrm{JH}$ has not yet been identified in other organisms, within the detection limits of our instrumentation. The real problems are that very few species have been extracted and examined in chemical detail, and that numerous plants and animals are already known to contain sesquiterpene like molecules which possess weak JH activity. To pursue this question, which bears on the environmental impact of $\mathrm{JH}$ analogs, new research would have to involve chemical identification rather than rely on bioassay, which formed the basis of reports Granett (1975) $\mathrm{JH}$ activity in mammalian tissues in the late 1950's. On phylogenetic grounds it could be anticipated that $\mathrm{JH}$ might occur in other classes of the phylum arthropoda, such as the crustaceans, which definitely contain the same molting hormones as insects Gilbert (1961). Even this is doubtful, because a large body of toxicological testing of IGRs on various crustacean species at several developmental stages has shown no effects which were even vaguely hormonal in nature. More extensive tests of the natural $\mathrm{JH}$ and several hundreds of their analogs revealed no hormonal activity on spider mites belonging to the class Acarina of the arthropod phylum, even though these phytophagous mites which are serious agricultural pests exhibit a complex metamorphosis comparable with that of insects (Staal, 1975). Although it is conceivable that crustacean and non-insect metamorphosis is regulated by known $\mathrm{JH}$, perhaps so tightly protein-bound as to elude detection, it is more reasonable to presume that molecules other than the known $\mathrm{JH}$ may be responsible for the regulation of metamorphosis of arthropods other than insects. Though several workers have searched for effects of insect $\mathrm{JH}$ on nematodes, no clearly hormonal effects have been demonstrated Hatakoshi (1998). The widespread distribution of substances identical with insect molting hormones has been well documented and reviewed Gelman (1995), but in contrast the insect $\mathrm{JH}$ appear to be quite restricted to the insects.

\section{Diacylhydrazines}

Dibenzoylhydrazine (DBH) (Diacylhydrazine) compounds are nonecdysteroidal activators of the ecdysone receptor complex [heterodimer 
of ecdysone receptor (EcR) and ultraspiracle/ retinoid-X-receptor (USP/RXR)] in insects. Their use as insecticides is based on their capacity to induce a premature and incomplete moult during which susceptible insects die from desiccation and starvation. DBH compounds can be applied to the field because of their favourable physicochemical and biological properties, such as high hydrophobicity, which allows the penetration of the cuticle and the midgut of the insects, and their slow clearance from the insect's body, resulting in persistence of action. In addition, DBHs have a high safety profile, which is compatible with their use in integrated pest management (IPM) programmes.

All commercial ecdysone agonists used in IPM are DBH analogues, such as tebufenozide (RH-5992), methoxyfenozide (RH-2485), halofenozide (RH-0345) and chromafenozide (ANS-118). The ecdysteroid agonist RH-0345 (halofenozide) represents a new class of IGRs, which has been developed to provide safe insecticides with high activity in Coleoptera. Agrochemical research has resulted in the discovery of novel insecticides that act on selective biochemical sites present in specific insect groups. Good examples are the insect growth regulators (IGRs), ecdysteroid agonists, which affect the hormonal regulation of moulting and development processes.

DBH compounds are considered to be highly specific to lepidopteran insects, although halofenozide is also marketed for control of coleopteran pests. According to in vitro reporter assays for activation of EcR/USP (RXR) in insect cell lines, activities of DBH compounds differ by orders of magnitude between lepidopteran versus coleopteran and dipteran insects. However, recent observations have indicated that in vivo parameters can play a large role in determining the efficacy of a compound, which cannot be predicted by the activity of the compounds in vitro reporter assays. Firstly, halofenozide was used successfully against soil-dwelling coleopterans, such as scarabid beetles, in spite of their rather low activity in binding assays using in vitro produced coleopteran EcR/USP (RXR) protein or reporter assays using coleopteran cell lines.

Secondly, it was observed that, while activities of DBH compounds in reporter assays were nearly identical using cell lines derived from the silkmoth Bombyxmori (Bm5 cells) and the cotton leafworm Spodopteralittoralis(S12 cells), great differences were observed in toxicity assays between larvae of these lepidopteran species.

Various studies revealed that the prototype ecdysteroid against RH-5849 presented high activity in species of Lepidoptera and Coleoptera, but were superseded later by $\mathrm{RH}-$ 5992, RH-2485 and RH-0345, which have enhanced activity against lepidopteran and coleopteran pest insects. The ecdysteroid against compounds exert their toxicity by binding to the nuclear ecdysteroid receptor EcR as does the natural insect moulting hormone, 20-hydroxyecdysone (20E).

\section{Moulting hormones}

The brain/molting hormone ecdysone initiates the molting process and induces metamorphosis.

\section{Precocenes}

The compounds which would antagonize the insect hormone activity and derange the insect's development are called as "Precocenes" because of their ability to induce precocious metamorphosis of the immature insects. The mode of action of precocenes seems to be the prevention of JH biosynthesis corpora allata, since application of exogenous $\mathrm{JH}$ can reverse their action. 
Some of the agricultural pests used for the insects growth regulator activity

\section{Spodopteralitura}

Spodopteralitura is also known as the Oriental leafworm moth, Cluster caterpillar, Cotton leafworm, Tobacco cutworm, Tropical armyworm, Taro caterpillar, Tobacco budworm, Rice cutworm, and Cotton Cutworm. This moth is found in Asia, with some specific problematic pest population reports occurring in Cambodia, Hong Kong, India, the Pacific islands, Guam, American Samoa, and Hawaii. In Australia, it is found in northern two thirds of the country. It is not established in the United States; however, it is a pest of national, regulatory concern. Based on the available geographic records of this moth, it is predicted that 48 Per cent of the U.S. will be suitable for survival of this moth. This pest is considered to be of concern from a regulatory perspective. It is believed to have potentially high economic impact in terms of its direct pest damage, trade implications, high outbreaks or problems on new hosts.

\section{Scientific classification}

Kingdom: Animalia

Phylum: Arthropoda

Class: Insecta

Order: Lepidoptera

Family: Noctuidae

Genus: Spodoptera

Species: Spodopteralitura

\section{Cotton bug (Dysdercuskoenigii)}

It is serious pest of cotton which infests cotton in all the cotton growing regions of India. The red cotton bug, Dysdercusis a most important damaging pest of okra also in North Eastern Hill region. Both the adults and nymphs feed on developing fruits of Cotton Gossypium hirsutum Linn. (Malvaceae), the major crop of India and grown on large scale in
Maharashtra, Gujarat, Karnataka, Madhya Pradesh, Punjab, Rajasthan, Haryana, Tamil Nadu and Uttar Pradesh. This crop is severely attacked by number of pests. Red cotton bug or cotton stainer or red seed bug is a colourful bug in the family pyrrhocoridae. Seriously affects the crop yield and quality of fruits thereby reducing the market value of cotton. Besides cotton, it feeds on variety of plants. Female $(20 \mathrm{~mm})$ is longer than male $(18 \mathrm{~mm})$.

\section{Scientific classification}

Phylum: Arthropoda

Subphylum: Uniramia

Class: Insecta

Order: Heteroptera

Family: Pyrrhocoridae

Subfamily: Pyrrhocorinae

Genus: Dysdercus

Species: Dysdercuskoenigii

\section{IGRS from neem}

Leaf and seed extracts of neem which contains azadirachtin as the active ingredient, when applied topically causes growth inhibition, malformation, mortality and reduced fecundity in insects.

\section{Hormone mimics from other living organisms}

Ecdysoids from plants (Phytoecdysones) have been reported from plants like mulberry, ferns and conifers. Juvenoids have been reported from yeast, fungi, bacteria, protozoans, higher animals and plants.

\section{Carvacrol}

Recent studies have indicated that Carvacrol has anti-hapatocarcinojenic activities; Hep G2 cell growth by inducing apoptosis as shown by Hoechst 33258 strain and flow cytometric (FCM) analysis. Apoptosis by direct activation of mitochondrial pathway and the mitogen 
activated protein kinase pathway has been suggested to play important role in antitumor effect of Carvacrol. The first report on identification of the antiproliferal effects of Carvacrol in prostate cancer cells has stated that the carvacrol may be valuable as the potential the therapeutic agents for prostate cancer. These are the importance of Carvacrol in Pest Management. Monoterpenoids can induce several types of biological activates in pests. They are relatively safer group of natural products. Monoterpenoids are ten carbon hydrocarbons or related compounds having hydroxyl, carbonyl, carboxylic functional groups which can be relatively easily modified to various derivatives. They are naturally occurring and exists abundantly in plants, marine algae and insects. More than 1000 Monoterpenoids have been identified so far and many possess interesting chemical ecological properties. They are typically rather more lipophillic compounds and thus may interfere with basic metabolic, biochemical, physiological \& behavioral functions of insects. They have shown toxicity to insects but most of them are less toxic to mammals. Several monoterpenoids are included on the generally recognized as safe (GRAS) list in U.S.A. and they are used as artificial flavouring agents in food, as fragrances in cosmetics and as pharmaceuticals.

As part of search for natural insecticides useful for fruit and vegetable plant protection, insecticidal activities of 23 water-distilled essential oils and their principal compounds including carvacrol have been reported by Sampson et al., From the outcome it has been suggested that aphids such as Turnip aphids that are resistant to conventional organophosphate insecticides and Lipaphis pseudobrassicae which is also vectors ca 10 non-persistent crucifer viruses can be controlled easily with them and essential oils and further noted with their novel, highly bioactive compounds can be developed as insecticides. Carvacrol had also been shown to have broad insecticidal and acaricidal activity against agricultural, stored-product, and medical arthropod pests.

\section{Carvacrol derivatives in pest management}

Series of Carvacryl ethers and esters have been synthesized and evaluated as antibacterial agents. Modifications into some of these derivatives exhibited potential for developing as antifungal and antibacterial agents. Synthetic derivatives of monoterpenoids have been reported as leads for new insect-control agents by Roller (1988). The results obtained through systematic bioassay and structure-activity relationships indicated that the monoterpenoids and their synthetic derivatives have a relatively wide spectrum of activity against agricultural and public health insect pests, the acyl derivatives of the monoterpenoids, has been reported to have significantly improved the acute, fumigant, larvicidal and ovicidal activities against insects. The ether derivatives exhibited enhanced insecticidal activity. Some of them exhibited insect growth and development activity against mosquito larvae. Our research group has emphasized mainly on the preparation characterization and biological activities of derivatives of the two phenolic monoterpenoids, Thymol and Carvacrol.

A piece of research work on Carvacrol is on ether and ester derivatives and includes antifungal, antibacterial activities on plant pathogenic microorganisms as well as insecticidal activities against stored grain pests. Carvacryl esters were found to be most active against plant pathogenic bacteria. Carvacryl acetate and carvacryl allyl ether and thymyl 4-methoxybenzylideneaniline exhibited comparable antibacterial activity with standard streptocyclin against A.radiobacter and P.solanacearum (Fig. 1-6). 
Fig.1 Classification of insect growth regulators

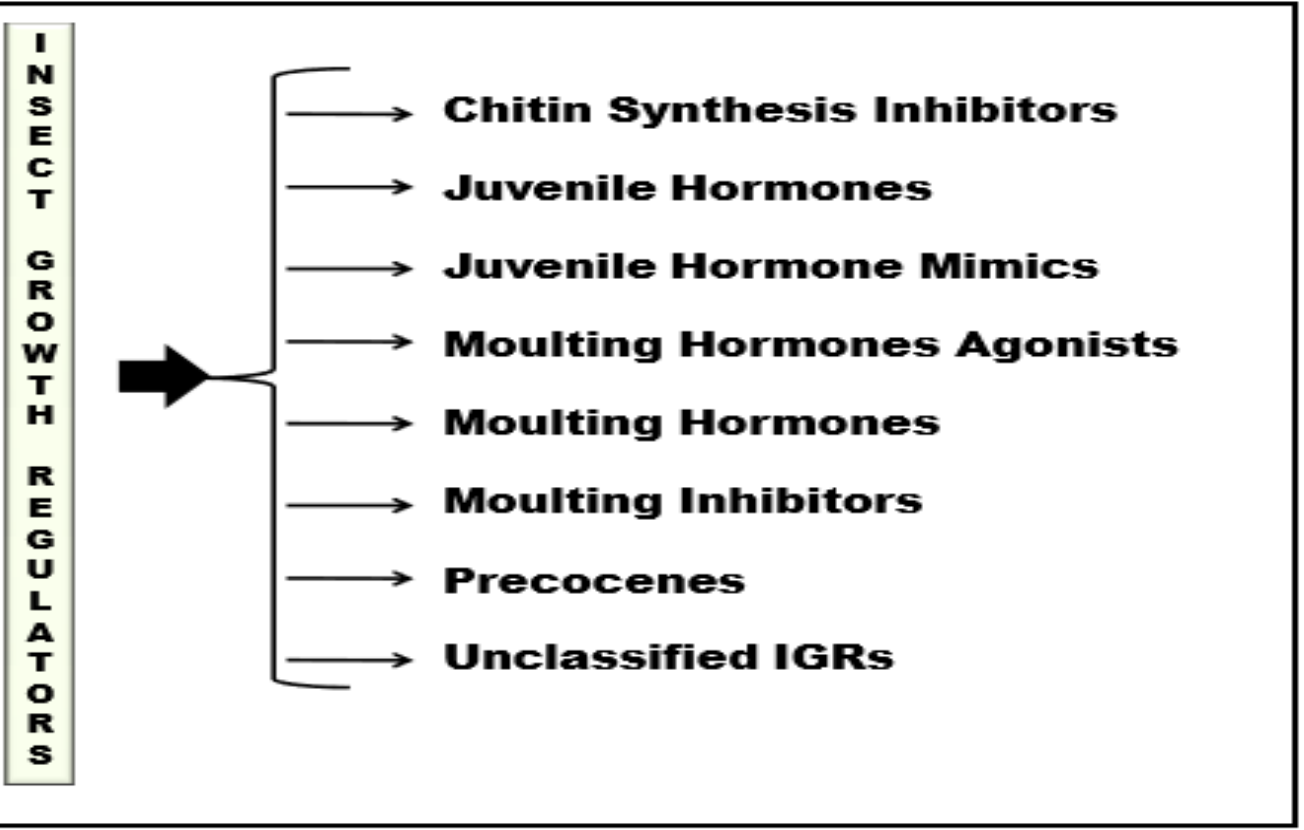

Fig.2 Market available chitin synthesis inhibitor / benzoyl phenyl urea compounds

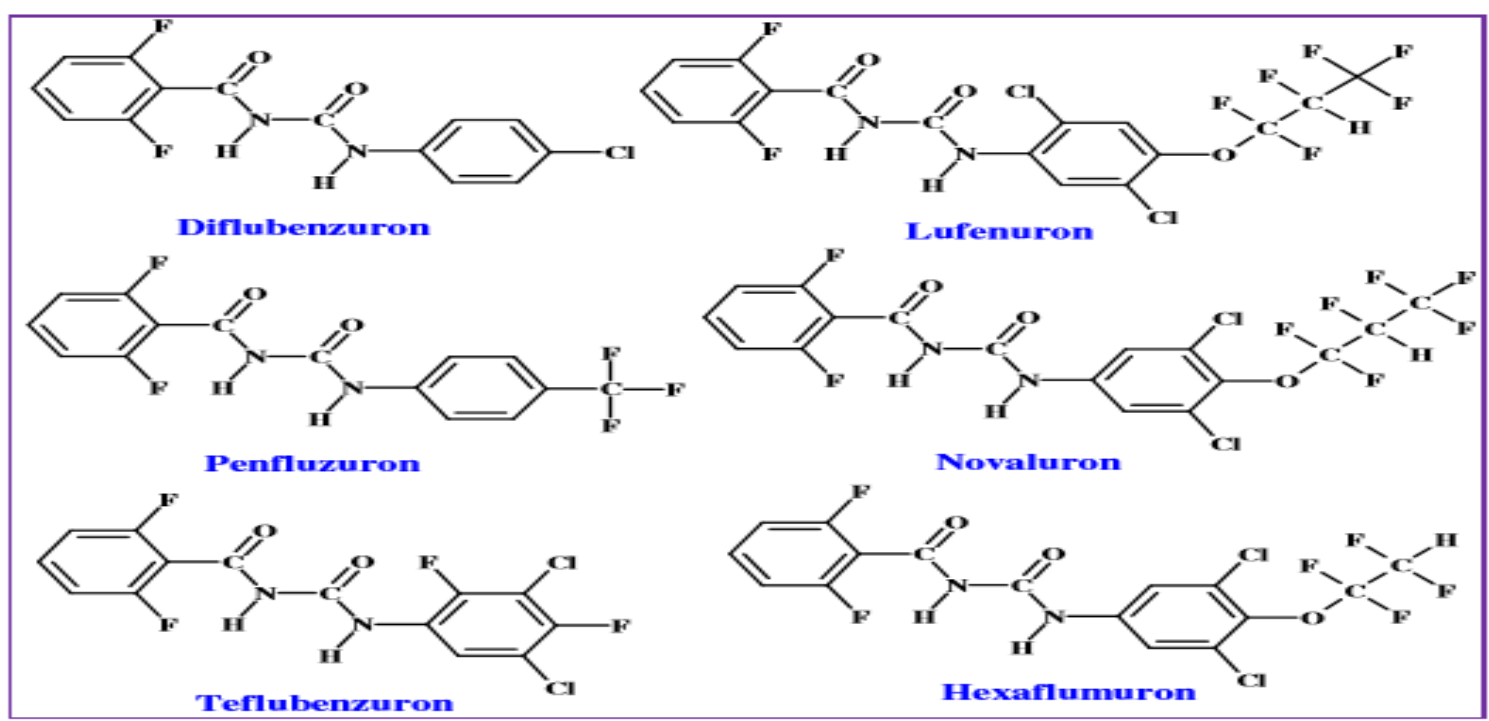


Fig.3 Juvenile Hormone I, II and III Compounds

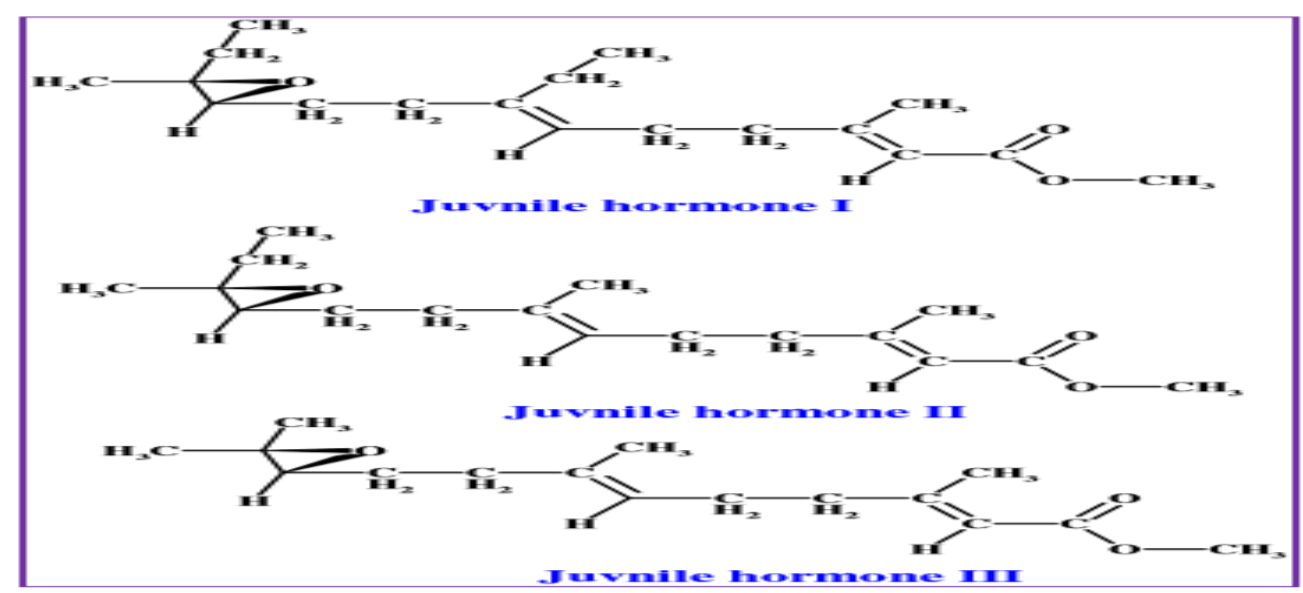

Fig.4 Diacylhydrazines as moulting hormone

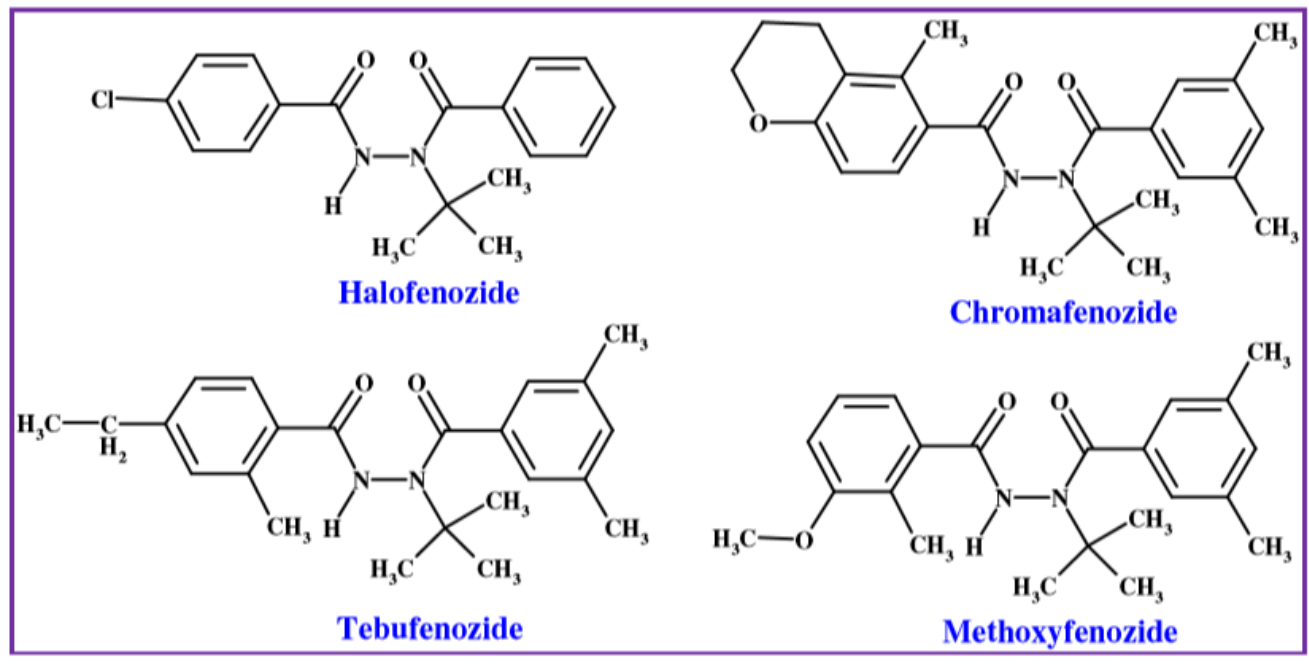

Fig.5 Moulting hormones $\alpha$-ecdysone and ecdysterone

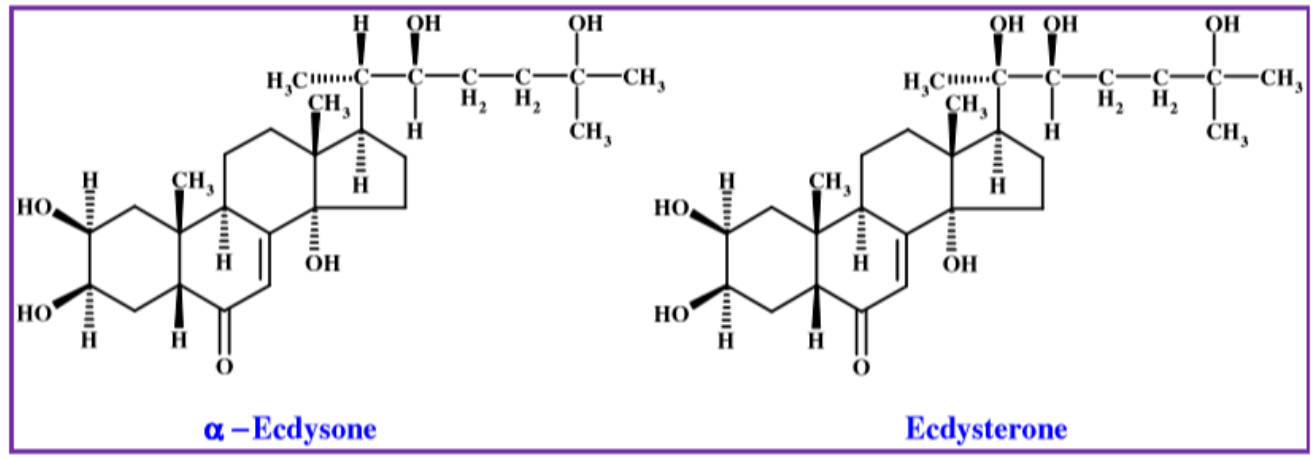


Fig.6 Precocenes I, II and III

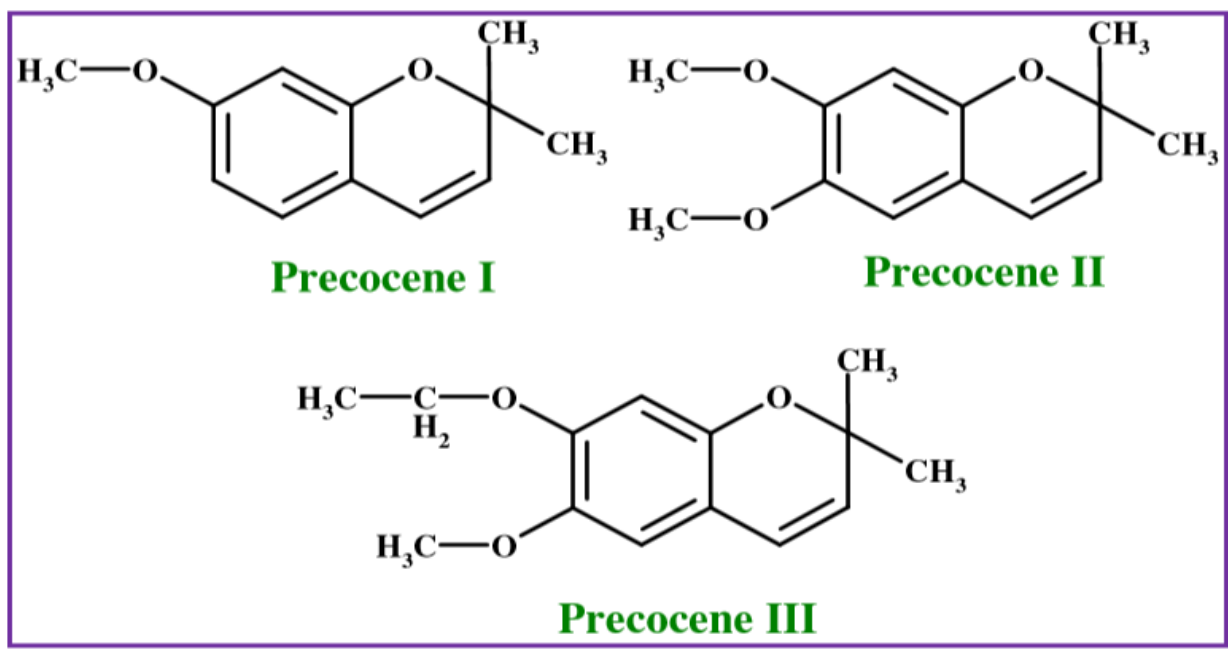

These are the advantages of using IGRs; effective in minute quantities and so are economical Target specific and so safe to natural enemies Bio-degradable, nonpersistent and non-polluting Non-toxic to humans, animals and plants. Most synthetic insecticides are toxic to all animals including human beings. Although many insecticides can be used safely, a few are persistent in the environment and a small number have multigenic, carcinogenic and teratogenic effects on human beings and domestic animals. Furthermore, their magnification in the food chain sometimes threatens non-target organisms. These facts have become of deep concern to agricultural and health scientists, producers and consumers alike. Based on the previous discussion, IGRs represent the newest of all approaches to operational and commercial insect control. Their species or stage-specificities that were higher than those of conventional insecticides offer a good alternative for a selective insect pest control that is in harmony with existing IPM programs. IGRs generally have a good margin of safety for most non-target biota including invertebrates, fish, birds, and other wildlife. They are relatively safe for human beings and domestic animals.

\section{References}

Ascher, K.R. 1993. Nonconventional insecticidal effects of pesticides available from the neem tree, Azadirachta indica. Arch. Insect Biochem. Physiol. 22: 433-449.

Asai, T.A., M. Kajihara, F. Fukada and S. Makekawa. 1985. Studies on the mode of action of buprofezin II. Effects on reproduction of the brown planthopper, NivaparvatalugensStal (Homoptera: Delphacidae). Appl. Entomol. Zool. 20: 111-117.

Awad, T.I., F. Önder and fi. Kısmalı. 1998. Azadirachtaindica A. Juss (Meliaceae) aøacındaneldeedilendođalpestisitlerüze rindebirinceleme. Türk. Entomol. Derg. 22: 225-240.

Bull, D.L. and R.W. Meola. 1993. Effect and fate of the insect growth regulator pyriproxyfen after application to the horn fly (Diptera: Muscidae). J. Econ. Entomol. 86: 1754-1760.

Carton, B., G. Smagghe and L. Tirry. 2003. Toxicity of two ecdysone agonists, halofenozide and metoxyfenozide, against the multicolored Asian lady beetle, Harmonia axyridis, (Col., 
Coccinallidae). J. App. Entomol. 127: 240-242.

De Wael, L., M. De Greef and O. Van Laere. 1995. Toxicity of pyriproxyfen and fenoxycarb to bumble bee brood using a new method for testing insect growth regulators. J. Apic. Res. 34: 38.

Gelman, D.B., R.A. Bell, A.B. DeMilo and J.P. Kochansky. 1995. Effect of KK-42 on growth, development, molting, and metamorphosis of the European corn borer, Ostrinia nubilalis (Hubner). Arch. Insect Biochem. Physiol. 28: 115.

Granett, J. and R.M. Wesoloh. 1975. Dimilin toxicity to the gypsy moth larval parasitoid, Apanteles melanoscelus. J. Econ. Entomol. 68: 577-580.

Hatakoshi, M., I. Nakayama and L.M. Riddiford. 1988. The induction of an imperfect supernumerary larval molt by juvenile-hormone analogs in Manduca sexta. J. Insect Physiol. 34: 373-378.

Liu M, Wang Y, Wangyang WZ, Liu F, Cui YL, Duan YS, Wang M, Liu SZ, Hui Rui NC, J. Agric. Food Chem. 2010, 58,6858 ,

Medina, P., G. Smagghe, F. Budia, P. Estal, L. Tirry and E. Vinuela. 2002. Significance of penetration, excretion, and transovarial uptake to toxicity of three insect growth regulators in predatory lacewing adults. Arch. Insect. Biochem. Physiol. 51: 91-101.

Rechcig JE, Rechcigl NA, Insect pest management: techniques for environmental protection. Lewis Publishers, New York, NY, 2000, 392.

Riddiford, L.M. and J.W. Truman. 1978. Biochemistry of Insect Hormones and Insect Growth Regulators, In: Biochemistry of Insects (Ed. M. Rockstein). Acad. Press, New york. pp. 307357.

Roller, H., and Dahm, K. H. The chemistry and biology of juvenile hormone. Recent Progr. Hormone Res. 24: 651 (1968).

Staal, G. B., 1975 insect growth regulator with juvenile hormone activity. Annual review entomology, 20, 417- 460.

Siddall, J.B. 1976. Insect growth regulators and insect control: A critical appraisal. Environ. Health Press.14: 119-126.

Wells, S.A., J. Immaraju, W.S. Ruggero and R. Nelson. 1993. Align, a new insect growth regulator that shows potential for control of cotton pests. Proc. Beltwide Cotton Conf., Memphis, TN. 1: 4344.

Wilkinson, J.D., K.D. Biever, C.M. Ignoffo, W.J. Pons, R.K. Morrison and R.S. Seay. 1978. Evaluation of diflubenzuron formulations on selected insect parasitoids and predators. J.Ga. Entomol. Soc. 13: 227-236.

Zhang, L., H. Kenji and S. Toshio. 1998. Cross-resistance to insect growth regulators in pyriproxyfen-resistant housefly. Appl. Entomol. Zool. 33: 195-197.

\section{How to cite this article:}

Sanjay Cyril Masih and Bhat Rayees Ahmad. 2019. Insect Growth Regulators for Insect Pest Control. Int.J.Curr.Microbiol.App.Sci. 8(12): 208-218. doi: https://doi.org/10.20546/ijcmas.2019.812.030 